\title{
Indirect-to-Direct Band Gap Crossover in Few- Layer Transition Metal Dichalcogenides: A Theoretical Prediction
}

\author{
Yajing Sun ${ }^{\dagger}$, Dong Wang ${ }^{*}$ and Zhigang Shuai ${ }^{*} \neq \S$ \\ ${ }^{\dagger}$ MOE Key Laboratory of Organic OptoElectronics and Molecular Engineering, Department of \\ Chemistry, Tsinghua University, Beijing 100084, PR China \\ ${ }^{\ddagger}$ Key Laboratory of Organic Solids, Beijing National Laboratory for Molecular Science (BNLMS), \\ Institute of Chemistry, Chinese Academy of Sciences, Beijing 10019o, PR China \\ ${ }^{\S}$ Collaborative Innovation Center of Chemistry for Energy Materials, Xiamen University, 351005 \\ Xiamen, PR China
}



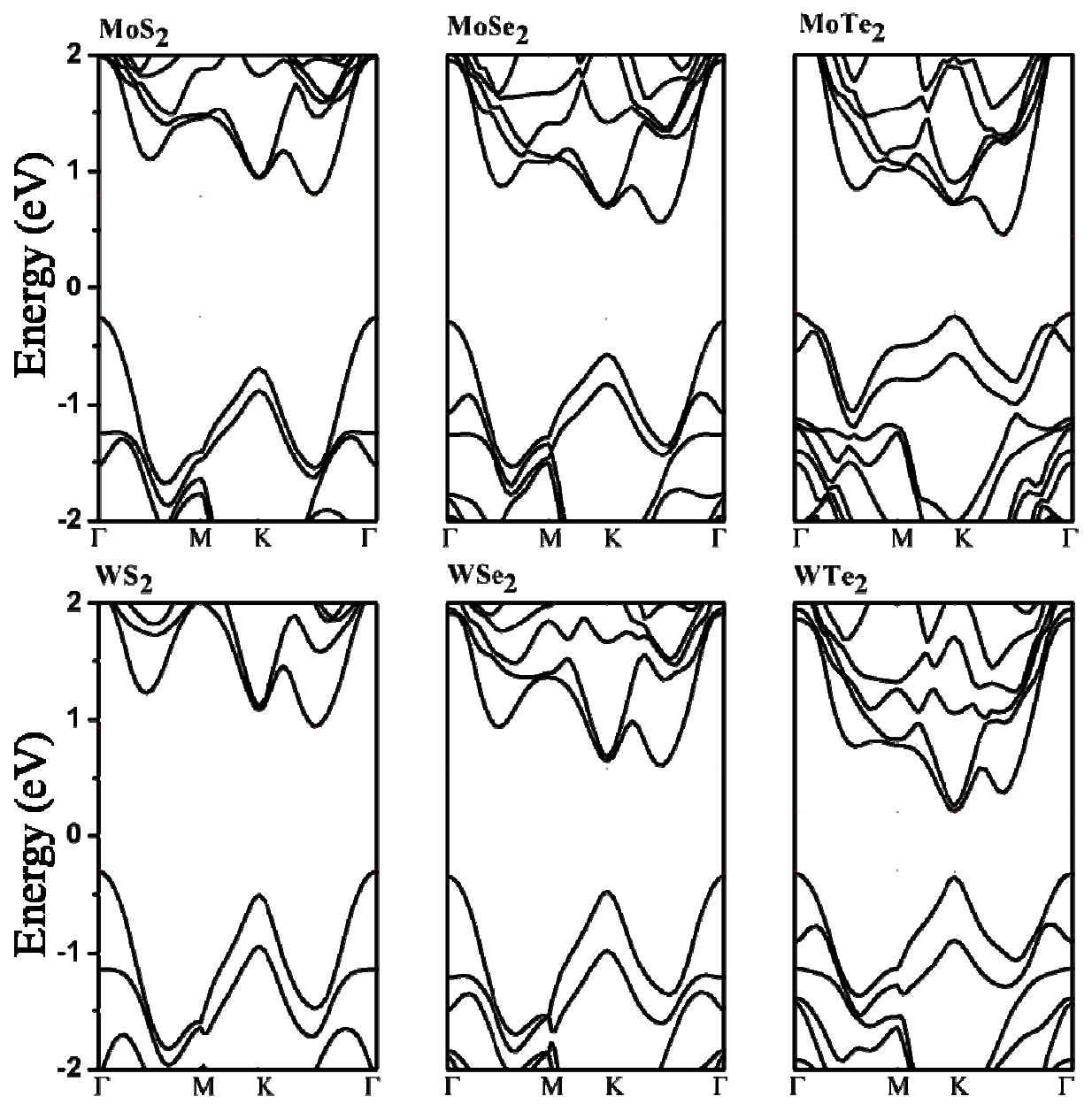

Figure $\mathrm{S}$. Band structures of bulk $\mathrm{MX}_{2}(\mathrm{M}=\mathrm{Mo}, \mathrm{W}$ and $\mathrm{X}=\mathrm{S}, \mathrm{Se}, \mathrm{Te})$.
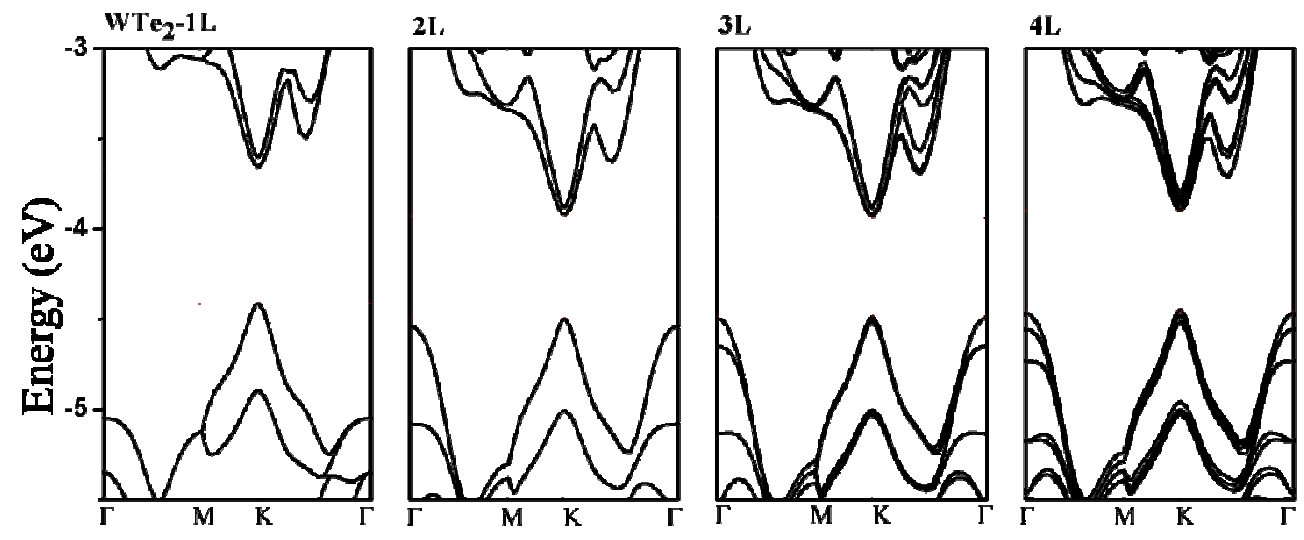

Figure $\mathrm{S}_{2}$. Band structure evolution of $\mathrm{WTe}_{2}$ with the layer thickness. 

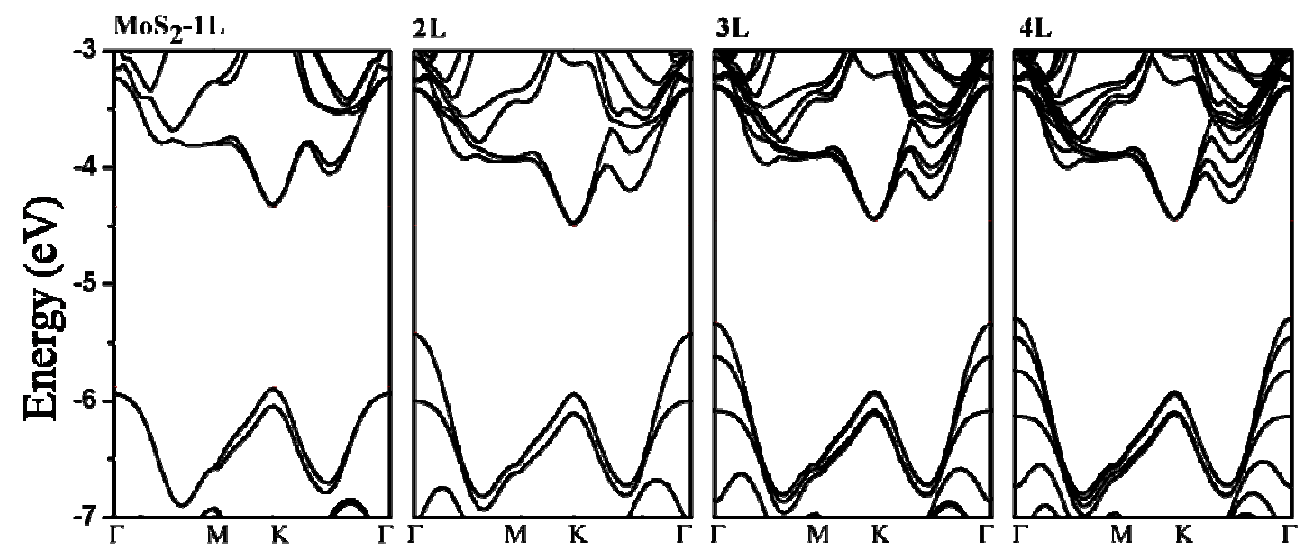

Figure $\mathrm{S}_{3}$. Band structure evolution of $\mathrm{MoS}_{2}$ with the layer thickness.
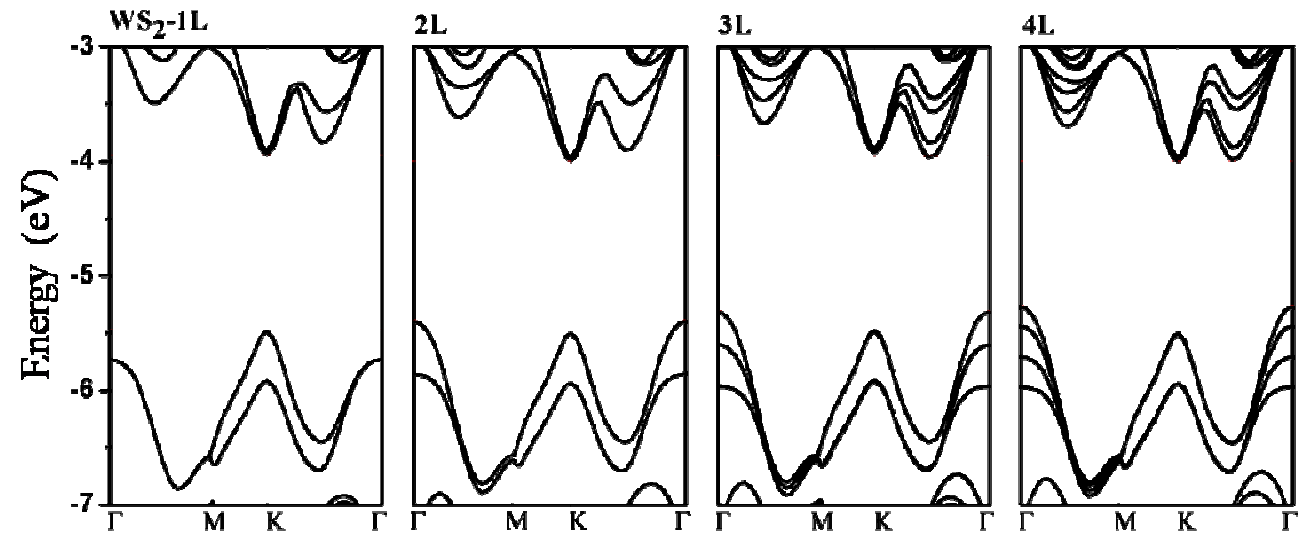

Figure $\mathrm{S}_{4}$. Band structure evolution of $\mathrm{WS}_{2}$ with the layer thickness. 


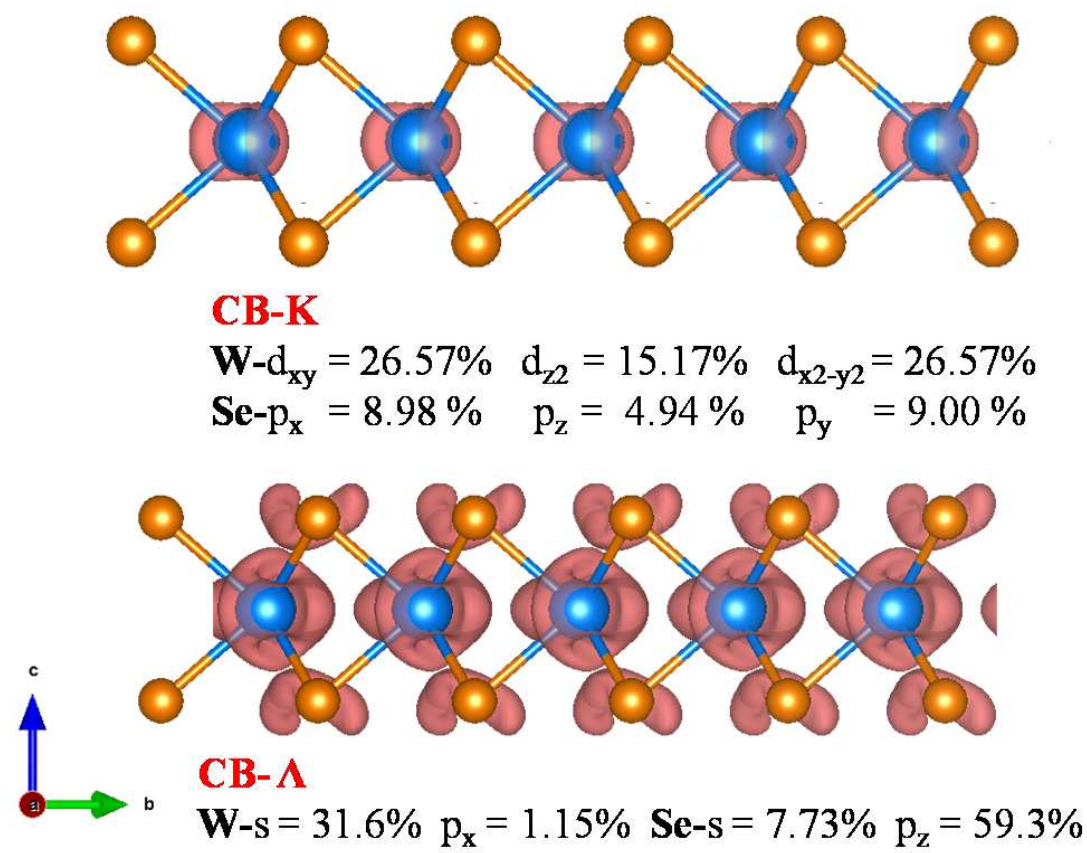

Figure S6. Charge density distribution of monolayer $\mathrm{WSe}_{2}$ at $\mathrm{K}$ and $\Lambda$ valleys of the conduction band for a contour value of $0.01 \mathrm{e} / \mathrm{bohr}^{3}$ and $0.03 \mathrm{e} / \mathrm{bohr}^{3}$ respectively.

Table Si. Structuralparameters and band gaps of monolayer, few-layer and bulk $\mathrm{MoS}_{2}, \mathrm{MoSe}_{2}$, $\mathrm{MoTe}_{2}, \mathrm{WS}_{2}$, and WTe .

\begin{tabular}{|c|c|c|c|c|c|c|c|c|}
\hline & & $\mathrm{a} / \mathbf{A}$ & $\mathbf{L}(\mathbf{X ~ X}) / \mathbf{A}$ & $\mathbf{H}(\mathbf{M ~ M}) / \mathbf{A}$ & $\mathbf{h}(\mathbf{X ~ X}) / \AA$ & Bond(M-X)/Å & Angle $(\mathrm{X}-\mathrm{M}-\mathrm{X}) \mathrm{/}^{\circ}$ & Band gap $(\mathrm{K}-\mathrm{K}, \Gamma-\Lambda) / \mathrm{eV}$ \\
\hline \multirow{5}{*}{$\mathrm{MoS}_{2}$} & $1 \mathrm{~L}$ & 3.184 & 3.126 & N.A. & N.A. & 2.413 & 80.75 & $1.596,1.947$ \\
\hline & $2 \mathbf{L}$ & 3.223 & 3.094 & 6.195 & 3.102 & 2.420 & 79.47 & $1.528,1.261$ \\
\hline & $3 \mathrm{~L}$ & 3.215 & 3.099 & 6.197 & 3.099 & 2.419 & 79.72 & $1.471,1.100$ \\
\hline & $4 \mathrm{~L}$ & 3.215 & 3.099 & 6.179 & 3.081 & 2.418 & 79.73 & $1.476,1.018$ \\
\hline & Bulk & 3.167 & 3.133 & 6.363 & 3.230 & 2.408 & 81.14 & $1.637,1.086$ \\
\hline \multirow{5}{*}{$\mathrm{MoSe}_{2}$} & $\mathbf{1 L}$ & 3.332 & 3.329 & N.A. & N.A. & 2.544 & 81.74 & $1.299,1.826$ \\
\hline & $2 \mathrm{~L}$ & 3.332 & 3.322 & 6.507 & 3.185 & 2.542 & 81.62 & $1.292,1.154$ \\
\hline & ${ }_{3} \mathbf{L}$ & 3.332 & 3.322 & 6.504 & 3.183 & 2.541 & 81.60 & $1.275,0.993$ \\
\hline & ${ }_{4} \mathrm{~L}$ & 3.332 & 3.322 & 6.505 & 3.183 & 2.541 & 81.60 & $1.275,0.933$ \\
\hline & Bulk & 3.332 & 3.346 & 6.654 & 3.307 & 2.542 & 81.64 & $1.270,0.860$ \\
\hline \multirow{2}{*}{$\mathrm{MoTe}_{2}$} & $\mathbf{I L}$ & 3.557 & 3.608 & N.A. & N.A. & 2.734 & 82.60 & $0.931,1.654$ \\
\hline & $2 \mathrm{~L}$ & 3.522 & 3.626 & 6.980 & 3.352 & 2.725 & 83.44 & $0.930,0.953$ \\
\hline
\end{tabular}




\begin{tabular}{|c|c|c|c|c|c|c|c|c|}
\hline & $3^{L}$ & 3.522 & 3.627 & 6.997 & 3.370 & 2.724 & 83.44 & $0.983,0.822$ \\
\hline & ${ }_{4} L$ & 3.522 & 3.625 & 6.983 & 3.358 & 2.723 & 83.40 & $0.982,0.765$ \\
\hline & Bulk & 3.521 & 3.625 & 6.990 & 3.365 & 2.723 & 83.44 & $0.966,0.686$ \\
\hline \multirow{5}{*}{$\mathrm{WS}_{2}$} & $\mathbf{I L}$ & 3.184 & 3.138 & N.A. & N.A. & 2.417 & 80.96 & $1.550,1.955$ \\
\hline & $2 \mathbf{L}$ & 3.194 & 3.131 & 6.508 & 3.376 & 2.419 & 80.66 & $1.510,1.543$ \\
\hline & $3^{\mathrm{L}}$ & 3.181 & 3.140 & 6.485 & 3.344 & 2.416 & 81.04 & $1.554,1.349$ \\
\hline & ${ }_{4} \mathrm{~L}$ & 3.194 & 3.131 & 6.510 & 3.378 & 2.419 & 80.68 & $1.506,1.332$ \\
\hline & Bulk & 3.174 & 3.145 & 6.589 & 3.444 & 2.415 & 81.26 & $1.586,1.247$ \\
\hline \multirow{5}{*}{$\mathrm{WTe}_{2}$} & $\mathbf{I L}$ & 3.550 & 3.629 & N.A. & N.A. & 2.737 & 83.01 & $0.756,1.394^{*}$ \\
\hline & $2 \mathrm{~L}$ & 3.622 & 3.573 & 7.018 & 3.441 & 2.752 & 81.01 & $0.574,0.616^{*}$ \\
\hline & $3^{\mathrm{L}}$ & 3.622 & 3.574 & 7.024 & 3.448 & 2.751 & 81.04 & $0.556,0.560^{*}$ \\
\hline & ${ }_{4} \mathrm{~L}$ & 3.608 & 3.581 & 7.026 & 3.442 & 2.747 & 81.36 & $0.548,0.568$ \\
\hline & Bulk & 3.562 & 3.630 & 7.298 & 3.668 & 2.743 & 82.86 & $0.587,0.565^{*}$ \\
\hline
\end{tabular}

${ }^{*}$ The indirect band gap transition is from $\Gamma$ to $\mathrm{K}$ for WTe2. 